\title{
Santiago, R. y Bergmann, J. (2018) Aprender al revés. Flipped Learning 3.0 y metodologías activas en el aula. Barcelona: Paidós Educación, pp. 240
}

\author{
Salvador Montaner-Villalba \\ UNED (España) \\ smontaner@invi.uned.es
}

Raúl Santiago y Jon Bergmann

\section{Aprender al revés}

PAIDOS Educación
En el prólogo, Mazur invita al docente a reflexionar en torno a aquello que sucede dentro del aula mediante el aprendizaje invertido. Plantea las funciones que desempeñan tanto el profesor como el alumnado dentro del aula. Este libro contiene diez capítulos. En el primer capítulo, Santiago y Bergmann ofrecen algunas consideraciones básicas en torno al aprendizaje activo. En el capítulo 2, los autores primero definen el flipped learning para, a continuación, relacionar el modelo flipped learning con la taxonomía de Bloom (1956), revisada por Anderson y Krathwol (2001). En tercer lugar, distinguen diferentes modalidades del flipped classroom (blended learning, PLE) y, finalmente, exponen las características del Flipped Learning 3.0 de forma bastante clara.

En el capítulo 3, los autores exponen los roles actuales del profesor flipped. Tras una concisa introducción en la que se invita al lector a reflexionar en torno a los nuevos roles que el docente del siglo XXI debe tener, Santiago y Bergmann asocian estos nuevos roles docentes con el modelo TPACK, desarrollado inicialmente por Koehler, Mishra y Cain (2015). Este modelo TPACK se resume en tres tipos de conocimientos: contenido, pedagógico y tecnológico, resultando la fusión de estos conocimientos en una gran diversidad de roles que el docente, según los autores del libro aquí reseñado, debe desempeñar. Algunas funciones son: el profesor debe ser experto en el contenido, eficiente planificador en cuanto al diseño de las unidades didácticas, el docente flipped debe ser un buen conferenciante, cercano y confiable, experto en el arte de preguntar, conocedor de la tecnología, debe ser un profesor creativo, etc. En segundo lugar, los autores reconocen tres tipos de profesores (creadores, neutrales y consumidores) para, por último, finalizar analizando primero algunas características generales sobre los docentes que ponen en práctica el modelo flipped learning en su trabajo diario y segundo como los docentes perciben el modelo flipped.

En el cuarto capítulo, los autores explican de forma concisa y clara las funciones que los alumnos del siglo XXI deben desempeñar. Para tal fin, los autores ofrecen aquí una doble perspectiva, es decir, determinan las funciones del alumnado flipped primero desde la propia experiencia de los propios docentes y segundo considerando las diversas opiniones que los alumnos han aportado mediante un cuestionario, dado que ellos son los protagonistas en el proceso de aprendizaje. Se presentan, a continuación, en este capítulo una serie de sugerencias que los docentes pueden ofrecer a los alumnos con respecto al modelo de clase inversa, a saber, estrategias para ver un video flipped, tomar notas, y mejorar la participación en clase.

En el capítulo cinco, los autores exponen una serie de pautas interesantes respecto a cómo diseñar las mejores prácticas para aplicar el flipped learning en el espacio individual, es decir, cuando los alumnos están trabajando de forma individual. En segundo lugar, se aborda la 
tecnología para llevar a cabo el aprendizaje invertido en el espacio individual, explicando así unos principios fundamentales con el objetivo de diseñar un buen contenido digital y, finalmente, analizan las competencias digitales más apropiadas para implementar el modelo flipped learning siguiendo los criterios del Marco Común de Competencia Digital Docente.

Santiago y Bergmann, en el capítulo 6, analizan las mejores prácticas en el espacio grupal con el fin de realizar un uso eficiente del tiempo en el aula. El enfoque de este capítulo consiste en resaltar una variedad de estrategias, tendencias y actividades a realizar en el espacio grupal con el alumnado para, luego, finalizar describiendo las metodologías más utilizadas por los docentes, tales como el Aprendizaje Basado en Proyectos, el Aprendizaje para el Dominio (Mastery Learning, en lengua inglesa), Aprendizaje Cooperativo, Gamificación, etc.

En el séptimo capítulo, se aborda qué dice la investigación en torno al modelo flipped learning, marcando las líneas maestras de todo aquello que se está realizando a nivel global. A este respecto, los autores se plantean si existe suficiente investigación sobre el flipped classroom. Tras unas breves consideraciones, determinan que sí hay un incremento notable de publicaciones empíricas desde el año 2012. Finalmente, explican una serie de pautas en torno a cómo aplicar en el aula flipped el modelo educativo investigación-acción.

En el capítulo 8, los autores ofrecen unas pautas respecto a cómo el docente puede mejorar el flipped classroom utilizando la analítica de datos así como la evaluación continua. Primero determinan con exactitud qué son las "analíticas del aprendizaje" (Amo y Santiago, 2017) para, posteriormente, analizar qué tipo de métricas se pueden analizar y su relación con la evaluación formativa. Santiago y Bergmann finalizan este capítulo exponiendo diversos ejemplos de analíticas de aprendizaje tanto en el espacio individual como en el grupal del modelo flipped, relacionándolos con diversas estrategias didácticas y herramientas digitales que son habitualmente usadas por los profesores.

En el capítulo nueve, presentan los autores varios consejos realmente útiles para mejorar el flipped classroom. Para tal fin, se exponen consejos y orientaciones que ofrecen en primera persona diversos profesores flipped que llevan ya tiempo aplicando este modelo en el aula. Algunos ejemplos son Domingo Chica, Alicia Díez, Eli Gómez, etc.

Finalmente, en el capítulo 10, Santiago y Bergmann analizan los factores que pueden favorecer ese "cambio" que posibilite el modelo de clase inversa tanto al conjunto de institución educativa como al profesorado, con un especial foco en diversos factores organizativos e institucionales relacionados con esos cambios que, en coherencia, van surgiendo. El presente libro está dirigido a todo aquel docente que tenga interés en aplicar el modelo flipped.

\section{REFERENCIAS BIBLIOGRAFÍCAS}

Amo, D. y Santiago, R. (2017) Learning Analytics. La narración del aprendizaje a través de los datos. Barcelona: Editorial UOC, pp. 216

Anderson, Lorin W.; Krathwohl, David R., (eds.) (2001). A taxonomy for learning, teaching, and assessing: A revision of Bloom's taxonomy of educational objectives. Allyn and Bacon.

Koehler, M.J.; Mishra, P. y Cain, W. (2015) ¿Qué son los saberes tecnológicos y pedagógicos del contenido (TPACK)?, Virtualidad, Educación y Ciencia, 10(6), 9-23

\footnotetext{
(c) $)(1)(2)$

Los textos publicados en esta revista están sujetos a una licencia de Reconocimiento 4.0 España de Creative Commons. Puede copiarlos, distribuirlos, comunicarlos públicamente y hacer obras derivadas siempre que reconozca los créditos de las obras (autoría, nombre de la revista, institución editora) de la manera especificada por los autores o por la revista. La licencia completa se puede consultar en:Licencia Creative Commons Atribución-NoComercial-Compartir por igual 4.0 Internacional.
} 